\title{
Pharmacists' Awareness of COVID-19 and Perceptions of their Roles, Barriers, and Roles of Policymakers: exploring the Middle East and North Africa (MENA)
}

\author{
Iman Basheti ${ }^{1}$, Feras Darwish El-Hajji ${ }^{1}$, Razan Nassar ${ }^{1}$, Samar Thiab ${ }^{1}$, Muna Barakat ${ }^{1}$, \\ Mariam Basheti ${ }^{2}$, Alberto Berardi ${ }^{1}$, and Bandana saini ${ }^{2}$ \\ ${ }^{1}$ Applied Science Private University \\ ${ }^{2}$ The University of Sydney
}

June 11, 2020

\begin{abstract}
Background: The coronavirus infection (COVID-19) was declared in January 2020 as a public health emergency of international concern. The Middle East and North Africa (MENA), like other parts of the world, suffered from several epidemics over the years. Pharmacists have vital roles to play to prevent the spread of this virus. Objectives: To assess the awareness of COVID-19 amongst pharmacists from countries located in the MENA countries, and to assess their perspectives of their role, barriers, and roles of the educational institutions and pharmaceutical associations in preparing them for their roles during the pandemic. Methods: An online survey was conducted to run a descriptive cross-sectional study in Jordan from 12th to 22 th April 2020. The questionnaire was validated and put on Facebook for pharmacists' recruitment and assessment of their awareness (20 questions with a score out of 20) about epidemics/pandemics and COVID-19, their perceived roles and barriers, and roles of the educational institutions and pharmaceutical associations. Data were analyzed using Statistical Package for the Social Science (SPSS). Results: Study participants $(\mathrm{n}=2589)$ had a mean age of $29.3(8.2)$ years and $1329(51.5 \%)$ were females. Most of the participants were from Egypt 819 (40.8\%), Followed by Jordan, Algeria, and Syria. As for the sources of information about coronavirus treatment among the study participants, $60.8 \%$ got their information from the social media. Fear as a consequence was identified by the majority of participants $(87.7 \%)$. The majority of pharmacists identified positive roles for the pharmaceutical association and pharmaceutical associations. Conclusion: Pharmacists from the MENA countries believe they got enough education previously about epidemics/pandemics, and the majority follow on the latest coronavirus updates from social media. Fear was reported as the major barrier that requires resolution by the policymakers. Certain gaps in the awareness about COVID-19 were identified.
\end{abstract}

\section{What is already known about this topic?}

The coronavirus infection (COVID-19) was declared in January 2020 as a public health emergency of international concern. The Middle East and North Africa (MENA), like other parts of the world, suffered from several epidemics over the years. Pharmacists have vital roles to play to prevent the spread of this virus. To prepare globally uniform pharmacy response directives for pandemics, pharmacists' awareness and perspectives around roles during pandemics in different regions of the world need to be assessed.

\section{What does this article add?}

This article adds that Pharmacists from the MENA countries (Egypt, Jordan, Syria, Palestine, Lebanon, and Iraq) believe they got enough education previously about epidemics/pandemics, and the majority follow on the latest coronavirus updates from social media. Fear was reported as the major barrier that requires resolution by the policymakers. Certain gaps in the awareness about COVID-19 were identified. In addition, 
a significant correlation between pharmacists' awareness scores across the study countries with the COVID-19 international mortality statistics was found.

Keywords: coronavirus; pandemics; pharmacists; educational institutions; Pharmaceutical association; Middle East; North Africa; MENA.

Abstract word count: 295; Manuscript word count: 3983

\section{Introduction}

According to the World Health Organization (WHO), a pandemic is the worldwide spread of a new disease ${ }^{1}$. Viral pandemics are caused by viruses that possess a surface protein to which the majority of people lack immunity, which then spreads between people within the population extending beyond national borders ${ }^{2}$. Historically, many viral pandemics have been documented. The most notorious of these is the Spanish influenza pandemic of 1918-1920, which was caused by the H1N1 virus and was responsible for about 40 million deaths worldwide ${ }^{3}$. Other pandemics include the Asian Influenza (1957) caused by the H2N2 virus, the Hong Kong Influenza (1968) caused by H3N2 virus², the Severe Acute Respiratory Syndrome (SARS) which occurred in 2003 and was caused by SARS coronavirus (SARS-CoV) ${ }^{4}$, and the Middle East respiratory syndrome (MERS) which occurred in 2012 and was caused by coronavirus (MERS-CoV) ${ }^{4}$. Recently, on the $31^{\text {st }}$ of December 2019, a case of pneumonia of unknown cause in Wuhan was reported to the WHO Country Office in China ${ }^{1}$. It was later identified as coronavirus disease-2019 (COVID-19) caused by the novel coronavirus officially called SARS-CoV-2, belonging to the family Coronaviridae ${ }^{5}$. The Coronavirus genome consists of a single strand positive sense ribonucleic acid (RNA) ${ }^{4}$. The virions (virus particles) are enclosed in a lipid envelope characterized by the presence of crown-like spikes on the surface which have led to the nomenclature -where 'corona' implies the crown like appearance created by these bulbous spikes. ${ }^{4}$. SARSCoV-2 usually causes mild symptoms such as fever, dry cough, and sore throat. However, it can sometimes lead to fatal complications such as severe pneumonia, pulmonary edema, acute respiratory distress syndrome (ARDS), organ failure and septic shock ${ }^{6}$. The spread of the virus to many countries around the world in a short space of time with no clear treatment or vaccine in sight as yet, is alarming and has resulted in high workload for healthcare teams globally ${ }^{7-9}$. Effective strategies currently followed to slow the spread of the virus included hygiene practices such as proper hand washing, hand sanitizing and wearing facemasks and gloves (personal protective equipment), in addition to social distancing and public quarantine measures ${ }^{10}$.

The Middle East and North Africa (MENA), like other parts of the world, have experienced several epidemics over the years. Outbreaks of plague, smallpox ${ }^{11}$, cholera ${ }^{12}$, and meningococcal infection ${ }^{13}$ have all been documented, especially in Saudi Arabia during the annual Pilgrimage (Hajj) as Muslims from more than eighty countries convene together, which led the Saudi authorities to require all visitors to immunize against certain diseases ${ }^{11,12}$.

Community pharmacists (or retail pharmacists as they are known in some countries) have a crucial role to play during pandemics ${ }^{14}$. One of the most important role of community pharmacists is to ensure the availability of effective therapy in addition to health education and health promotion activities and vaccination provision ${ }^{15-17}$. Moreover, community pharmacists need to engage with patients, providing them with counseling regarding the safety precautions to minimize exposure and infection probabilities as well as psychological and mental health support during a pandemic. Pharmacists would be expected to support general health care teams in infection prevention, as well as alerting public health officials of potential outbreaks/cases ${ }^{18,19}$. During pandemics pharmacists' role in managing minor ailments increases as doctors and hospitals become overwhelmed ${ }^{18,19}$. The International Pharmaceutical Federation (FIP) released a guideline during March 2020 stating the needed information for pharmacists and the pharmacy workforce to help them in dealing with the COVID-19 outbreak ${ }^{20}$, and acknowledging the responsibilities put upon the pharmacists in the control of the COVID-19 outbreak ${ }^{20}$. This mandate highlights the clear need for pharmacists to convey factual evidence based and up-to-date information to the public ${ }^{21 .}$ In a study conducted during the pandemic outbreak of H1N1, it was found that patients rely on information provided by pharmacists and considered them an effective educational source regarding the pandemic in addition to 
trusting pharmacists as immunizers ${ }^{22}$. In previous 'pandemic preparedness testing studies', in the United States (US), community pharmacists have expressed concerns that need to be considered by policymakers, for example, about critical medicine shortages or managing medicine hoarding ${ }^{17}$. Collating published literature, it appears that there are various research studies published to guide pharmacists on how to improve patient care services and outcome ${ }^{19,23,24}$, however, pharmacists are still in need for more tailored and extensive training to improve preparedness for emergency situations ${ }^{18}$. To prepare globally uniform pharmacy response directives for pandemics, pharmacists' awareness and perspectives around roles during pandemics in different regions of the world need to be assessed. In light of the repeated pandemic/epidemic experiences in the MENA region, the aim of this study, therefore, was to assess awareness of COVID-19 amongst pharmacists from countries located in the MENA and to assess their perspectives of their role, barriers, and roles of the educational institutions and pharmaceutical associations in preparing them to be able to deal with the pandemic.

\section{Method}

\section{Study design and participants}

This study was conducted from the $12^{\text {th }}$ of April to the $22^{\text {th }}$ of April 2020 over 10 days during the COVID-19 outbreak and public quarantine in most of the MENA countries. A descriptive cross-sectional study design (an online survey) was used to address the study objectives. The research team developed an online survey (based on the current information regarding the COVID-19) in order to meet the study objectives which included assessing MENA region pharmacists':

a) readiness to combat any epidemic/pandemic with a focus on the COVID-19 pandemic, assessing their clinical awareness, and b) views on the role of the pharmacy educators/educational institutes/pharmaceutical associations in preparing future pharmacists to deal with epidemics/pandemics and the COVID-19 specifically.

Any licensed pharmacist or enrolled pharmacy student in the MENA countries (community or hospital pharmacist, academic, industry based pharmacist, etc.) was deemed eligible for participation in the study. No risk was posed to the participants' and their participation was voluntary. The research team considered completing the survey as informed consent by pharmacists for participating in this study. Ethics approval was obtained from the Faculty of Pharmacy, Applied Science Private University, Jordan.

\section{Survey Development}

An extensive review of the literature was conducted to inform the development of items within this survey ${ }^{14}$. The survey was administered in the Arabic language (common across the MENA region). A variety of questions relevant to the study objectives, were also sourced from many avenues the research team had (e.g. FIP/WHO documents). The research team revised the survey in order to eliminate duplicates and refine question. The team further checked for local context and the aptness of language (slight regional variations in nuanced meanings of words).

In order to ensure survey face validity, six expert academics in Pharmacy Practice and Education were requested to evaluate the first draft. A pretest for the survey was conducted and necessary refinements were made. The pretest included evaluating the survey from different aspects such as the wording and the clarity of the questions and whether each question is relevant for inclusion with respect to the study objectives. As a final point in the survey development, the research team re-examined each question and made sure that the survey was suitable for online administration (relevance, clarity, context, response categories, format and layout).

The final version of the survey was organized into five main sections that address several topics of interest. The first sectionincluded items aimed at collecting participant's demographic data. The latter four sections included several items relevant to the study objectives (Figure 1).

Countries of the participants were grouped into geographic clusters (regions) that are: East Mediterranean 
countries and Iraq, Egypt, North Africa, Gulf countries, and other Arab countries.

\section{Survey Implementation}

Social media (Facebook and WhatsApp) was used to recruit the participants. The survey questions could be completed within an average of 10 minutes. Participants were able to view the ethics committee approval and the information about the purpose of the survey and what participation entailed before completing the survey.

\section{Statistics of confirmed cases and deaths}

The COVID-19 statistics of cases and deaths announced at the beginning and around the end of the study period (12 $2^{\text {th }}$ to $22^{\text {th }}$ of April 2020) for the countries which had at least one confirmed case at the beginning of the study were collected from the WHO Coronavirus Disease (COVID-19) Dashboard ${ }^{25}$.

\section{Sample size}

Sample size calculation was performed using the following formula: $\mathrm{n}=\mathrm{P} \times(100-\mathrm{P}) \times \mathrm{z} 2 / \mathrm{d} 2$. Where $\mathrm{P}$ is the anticipated awareness score percent, $\mathrm{d}$ is the desired precision, $\mathrm{z}$ is the appropriate value from the normal distribution for the desired confidence interval. Using $95 \%$ confidence levels, $5 \%$ precision level and an anticipated $50 \%$ proportion of participants receiving an appropriate score (this conservative value results in the highest possible sample size that can be used in this study, as no previous studies in this area where found to indicate level of awareness), a sample size of 385 was required.

\section{Statistical analysis}

Following data collection, the survey responses were coded and entered into a customized database using the Statistical Package for the Social Sciences (SPSS), Version 24.0 (IBM Corp., Armonk, New York, USA). Descriptive results were presented as means and standard deviations for continuous variables and percentages for qualitative variables. A one way ANOVA test was performed to analyse regional differences in perception scores. All tests were two-tailed. A P-value of $<0.05$ was considered statistically significant.

Correlation between awareness score (out of 20) and the COVID-19 statistics of cases and deaths announced at the beginning and end of the study period $\left(12^{\text {th }}\right.$ to $22^{\text {th }}$ of April 2020) for the countries which had at least one case at the beginning of the study was also conducted.

Linear regression was used to screen for the factors affecting participants' awareness score about coronavirus pandemic versus chosen independent variables in the study, i.e. age, area of residency (city and urban areas or rural areas), country, region, having children, educational level, university type (the university where participants had studied and/or are studying at; public versus private), years of experience, number of professional education workshops attended during the last year, work setting, source of previous knowledge about epidemics and pandemics, source of updates about COVID-19 management, and current satisfaction with knowledge about COVID-19. These predictor measures (independent variables) were considered as candidates for linear regression modelling if they had a significance value $\mathrm{p}$ [?]0.25 in univariate analyses. The candidate variables were subjected to backward linear regression, where finally only the significant variables (i.e. p [?]0.05) were retained with the model equation constant. Variables were selected after checking their independence, where tolerance values $>0.1$ and Variance Inflation Factor (VIF) values were $<10$ were selected to indicate the absence of multicollinearity between the independent variables in regression analysis. The homoscedasticity assumption for multiple linear regression was checked using Breusch-Pagan test, with a $\mathrm{p}[?] 0.05$ indicating the absence of heteroscedasticity.

\section{Results}

Study participants $(\mathrm{n}=2,589)$ had a mean age of 29.3 years and more than half $(51.5 \%)$ were females (Table 1). Most of the participants were from Egypt $(\mathrm{n}=819$ 40.8\%), followed by East Mediterranean countries (Jordan, Syria, Palestine, Lebanon) and Iraq. More than $80 \%$ of the participants lived in urban areas (including capital cities). About $75 \%$ of the participants had, as a highest level of pharmacy education, either 
a bachelor degree in pharmacy or Pharm. D., while the rest were either pharmacy students or technicians, or they had received other post-graduate degrees. The majority were alumni of public universities (75.7\%). More than half of the participants were practicing pharmacy either in community pharmacy or in hospital, years of work-experience tended to be short, i.e. less than and up to 5 years, and number of attended professional development workshops was low on average.

While only $30 \%$ of the participants had received enough education about epidemics/ pandemics and updates about coronavirus treatment, $91.2 \%$ of them do follow the latest corona virus updates on the treatment. Results were almost similar across all regions (Figure 2). As for the sources of information about coronavirus treatment among the study participants, $60.8 \%$ reported that they sourced their information from social media, followed by WHO reports and published articles (Figure 3).

Awareness scores (out of 20) were very similar across regions (Table 2), with a mean of 13.9 (out of a maximum of 20), no statistically significant difference was found between the regions $(\mathrm{n}=2589, \mathrm{p}=0.193$, one way ANOVA). A significant correlation between awareness scores across study countries was found $(\mathrm{p}=$ 0.026 , Pearson correlation $=-0.054)$ with the COVID-19 mortality statistics as they stood on the 15th of March 2020 for the countries which had at least one case at the beginning of the study ${ }^{25}$. Awareness score was higher in countries with lower mortality. No significant correlation was noted with other statistics of COVID-19 cases and deaths as they stood 15th of March, and 1st and 15th of April 2020.

Table 3 presents the total percentage and percentage of participants from the participating countries answering each of the awareness statements correctly. Interestingly, univariate analysis of the awareness score versus chosen independent variables showed significant association with many variables. This was reflected in the linear regression results, where older age participants, graduates of public universities versus private universities, higher annual attendance rates at professional workshops yearly, participants who practice in community pharmacy and hospital settings, participants who reported to have had previous knowledge from their graduating institute, participants who sourced their information about COVID-19from published studies, and those who were highly satisfied with their knowledge after 4 months of COVID-19 had higher knowledge scores (Table 4).

More than $90 \%$ of participants agreed with the statements provided regarding the role of the faculties/educational institutes expect for the statement 'your faculty has a role in preparing you to deal with any epidemic/pandemic' (74.8\%). As for the role of the pharmaceutical associations, more than $90 \%$ of pharmacists agreed with the statements provided except for 'pharmacists associations and societies have a role in preparing you to deal with epidemics/pandemics such as the coronavirus'. More than $80 \%$ of participants agreed with the statements provided regarding their role as pharmacists with the highest percentage being about the statement 'you ensure your personal safety by wearing gloves and masks and avoid close contact with patients'. Significant differences were seen regarding the regional distribution of study participants' perceptions about the current role of the faculties of pharmacies/educational institutes with regards to dealing with epidemics/pandemics and coronavirus specifically $(n=1998)$, while no significant differences were seen with regards to perceptions about the current role of pharmacists' associations and societies and current role of pharmacists (Figure 4, Table 5).

Most pharmacists believed that working in the pharmacy increases their fears about getting infected with coronavirus $(n=2131,82.3 \%$ strongly agree/agree). Significant differences found between regions $(\mathrm{p}=0.009)$. In addition, participants believed that they should receive training programs on how to provide mental health support for people during epidemic and pandemic outbreaks $(n=2257,87.7 \%$ strongly agree/agree $)$ with no significant difference found between the regions $(\mathrm{p}=0.426)$.

\section{Discussion}

Nowadays, the world is facing a predatory pandemic infection caused by COVID-19 requiring a concerted effort by health care professionals and public health experts to contain the rate of infection and the havoc it wreaks. Pharmacists, as essential health service providers, are important in the management and control of COVID-19. Hearteningly, the results of this survey indicated that pharmacists in the MENA countries have 
good awareness about the COVID-19 and acknowledge the different roles they can play during these times. Further, this study revealed that pharmacists are aware of the important responsibilities of the different educational faculties/educational institutes and pharmaceutical associations in preparing them to take on their roles during pandemics. Barriers impeding pharmacists from acting to their full potential were also explored, with fear from contracting the virus while performing their duty emerging as an important barrier that requires resolution.

The source of information gathered by healthcare professionals is important as it can affect clinical practice. The results of this study illustrated that more than $90 \%$ of the pharmacists monitor closely COVID-19 news, with the major sources of information being social media, WHO reports, and published literature. The collective responses are indicative of a well-informed group of pharmacists, possibly the high proportion of students and early pharmacy graduates contributed to this result. Although the pre-mentioned sources of information are important, Garcia and Julvez reported that referring to websites prepared by official public health organizations is important to ensure a trusted and updated source of information about COVID$19^{26}$. Interestingly, the majority of respondents reported that they have good awareness of COVID-19, and they have sufficient information about the infection. Social media may not always be the best source of accurate information although previous studies in the region indicate that this has been an important source of information for pharmacists in the MENA region through several epidemics earlier ${ }^{27}$.

Many medications for the management and treatment of the coronavirus have been discussed within the media/social media. Chloroquine and hydroxychloroquine for example have been frequently mentioned as potential treatments for the virus. In this study, $35 \%$ of the pharmacists reported that these medications can be used not only for treatment but for the prevention of the virus. Given that there is no evidence supporting their use, this belief is disturbing. Currently more than 140 clinical trials to test the COVID-19 efficacy of these medications alone or in combination with other medications, such as the antibiotic azithromycin, have been registered worldwide to explore their use as treatment/prevention options for COVID-1928. However, on the $24^{\text {th }}$ of April 2020, the United States Food and Drug Administration (FDA) issued a warning on the use of chloroquine and hydroxychloroquine for treating COVID-1929. The warning was due to reports of serious cardiac rhythm problems associated with the use of these medications particularly in combination with azithromycin, causing QT interval prolongation, a measure of delayed ventricular repolarization ${ }^{30}$. As another example, the majority of the study participants concurred that the use of non-steroidal antiinflammatory medications (NSAIDs) such as ibuprofen would increase the risk of COVID-19 complications. This probably was based on the fact that NSAIDs use as antipyretics for acute viral infections may induce serious complications such as empyema (presence of pleural effusion on chest radiograph and acidic pleural fluid) ${ }^{31}$. However, Capuano and co-workers published a review on the effect of NSAIDs on COVID-19 infection based on the available preclinical and clinical published studies ${ }^{32}$. They reported that there is conflicting data in the current literature, and there is no scientific evidence correlating NSAIDs use to the deterioration of COVID-19 patients' symptoms ${ }^{32}$. On March $18^{\text {th }}$ 2020, the European Medicines Agency (EMA) declared the need for further epidemiological studies for this issue, and urged health professionals to wait for formal results of an ongoing revision by the Pharmacovigilance Risk Assessment Committee (PRAC) on the association of Ibuprofen/Ketoprofen and worsening of infections ${ }^{33}$. Accordingly, NSAIDs usage by COVID-19 patients should be conducted with ultimate caution and under medical supervision. This conflicting news lead to conflicting believes and incorrect counseling provided by healthcare professionals all over the world, not just the MENA countries. Unifying the source of information for pharmacists worldwide and keeping it updated is vital to prevent such undesirable consequences.

Fear is a normal consequence to pandemics and public quarantine ${ }^{34}$. Our study findings demonstrated that the majority of pharmacists have fears about being infected with the coronavirus during their work at the pharmacy. Such findings have been acknowledged previously by pharmacists from other countries around the world ${ }^{35}$. Mental health is a priority for healthcare workers during pandemics, and pharmacists are not an exception ${ }^{35}$. Training sessions and online workshops were requested by the pharmacists in this study, as has been the case in previously published findings. In this term, the FIP had published a guideline for the pharmacists and pharmacy workforce, clarifying all the required responsibilities and role of both the 
community and hospital pharmacists ${ }^{36}$. This guideline also demonstrates all the preventive measures, tips on infection control and some recommendations for outpatient care $^{36}$. Interestingly, this study resonates with research investigating the experiences of other healthcare professionals, which highlights the need for more tailored and appropriate training to improve preparedness for deployment ${ }^{18}$.

Delivering medication to patients' homes can decrease infection spread especially for elderly patients were the risk of infection is increased. As an example, in Jordan, the Jordan Food and Drug Administration (JFDA) allowed community pharmacists and hospitals to provide free delivery of medications to patients' homes. Before the COVID-19, this practice was not allowed by the local laws ${ }^{37}$, which is the case in most countries in the MENA region. The majority of participants in this study were willing to deliver medicines to patients' homes. This provides an example of an effective service that can be successfully delivered everywhere if the safety of pharmacists from COVID-19 was guaranteed.

Social media was employed in the conduct of this study, which facilitated a large and rapid collection of sample size from numerous countries in the MENA region. It is of note that a significant correlation between pharmacists' awareness across the countries included in this study and the COVID-19 statistics of cases and deaths was found. This may indicate that countries with people (hence pharmacists) of higher awareness are likely to end with lower numbers of COVID-19 cases.

\section{Limitations}

The fact that this study was conducted via an online survey brings limitations just as it brought strengths. Such sampling technique led to a convenience sample which is a type of non-probability sampling that allows for data collection from a group of people easy to contact and/or reach ${ }^{38}$. Such approach may introduce sampling bias, as pharmacists who use social media more frequently had a better chance to participate in this study, giving results which may not be representative of all of the pharmacists groups in the MENA countries ${ }^{39}$. This might explain the relatively young age of the study participants, as men and older individuals are generally known to be underrepresented on social media ${ }^{39}$. Pharmacy students who participated in this study did not report their year of study, which can unveil useful information if indicated. Fifth-year students for example are expected to have different awareness levels compared to first and second-year students. Finally, although the survey was not completely validated, the items incorporated in it were done in real-time, based on the literature and reviews of a team of clinical pharmacy specialists.

\section{Conclusion}

Pharmacists from the MENA countries believed that they got enough education previously about epidemics/pandemics, and the majority follow on the latest coronavirus updates on treatments mostly from social media followed by the World Health Organization reports and published literature. The majority believed they have a major role in the management of the COVID-19, identifying fear as a barrier that requires resolutions by the policymakers. Certain gaps in the awareness about COVID-19 were identified, mainly concerning the conflicting news about Hydroxychloroquine and Ibuprofen use by infected patients. Such findings highlighted areas of improvement, and indicated that a significant association is found between higher awareness amongst pharmacists and the number of cases of COVID-19 patients in the MENA countries involved in the study. Results of this study have important international applicability, as pharmacists all over the world share similar barriers, responsibilities and engagements when it comes to the COVID-19 pandemic. Countries in the MENA region and worldwide share similar undergraduate pharmacy education and training, in addition to similar healthcare systems ${ }^{40}$, hence perspectives presented by the thousands of pharmacists involved in this study are an important springboard for further action.

Funding: This study was funded by the Deanship of Academic Research at Applied Science Private University, Amman, Jordan.

Conflicts of interest: None.

Specific contributions of each contributor to the paper: All authors were involved in all parts of study and manuscript preparation including literature search, study design, analysis of data, manuscript 
preparation, and review of manuscript.

\section{References}

1. World Health Organization (WHO): What is a pandemic? Accessed on 2-2010). Found at: https://www.who.int/csr/disease/swineflu/frequently_asked_questions/pandemic/en/.

2. Horimoto T, Kawaoka Y. Influenza: lessons from past pandemics, warnings from current incidents. Nat Rev Microbiol. 2005;3:591-600. doi: 510.1038/nrmicro1208.

3. Morens DM, Taubenberger JK. The Mother of All Pandemics Is 100 Years Old (and Going Strong)! Am J Public Health. 2018;108:1449-1454. doi: 1410.2105/AJPH.2018.304631. Epub 302018 Sep 304625.

4. Yang Y, Peng F, Wang R, et al. The deadly coronaviruses: The 2003 SARS pandemic and the 2020 novel coronavirus epidemic in China. J Autoimmun.2020;109:102434.:10.1016/j.jaut.2020.102434. Epub 102020 Mar 102433.

5. World Health Organization (WHO): Rolling updates on coronavirus disease (COVID-19). Accessed on 52020. Found at: https://www.who.int/emergencies/diseases/novel-coronavirus-2019/events-as-they-happen.

6. Sohrabi C, Alsafi Z, O'Neill N, et al. Corrigendum to "World Health Organization declares Global Emergency: A review of the 2019 Novel Coronavirus (COVID-19)" [Int. J. Surg. 76 (2020) 71-76]. Int J Surg. 2020;15:036.

7. Huang C, Wang Y, Li X, et al. Clinical features of patients infected with 2019 novel coronavirus in Wuhan, China. Lancet. 2020;395:497-506.

8. Kandel N, Chungong S, Omaar A, Xing J. Health security capacities in the context of COVID-19 outbreak: an analysis of International Health Regulations annual report data from 182 countries. The Lancet.

9. Huang C, Wang Y, Li X, et al. Clinical features of patients infected with 2019 novel coronavirus in Wuhan, China. The Lancet. 2020;395:497-506.

10. World Health Organization. Coronavirus disease (COVID-19) advice for the public. Accessed on 5-2020. Found at https://www.who.int/emergencies/diseases/novel-coronavirus-2019/advice-for-public.

11. Corkill NL. Epidemic smallpox in Jiddah; treatment with antibiotics. Br Med J.1951;2:663-667. doi: $610.1136 /$ bmj.1132.4732.1663.

12. Scrimgeour EM. Epidemic infections and their relevance to the Gulf and other Arabian Peninsula countries. J Sci Res Med Sci. 2003;5:1-4.

13. Memish ZA, Venkatesh S, Ahmed QA. Travel epidemiology: the Saudi perspective. Int J Antimicrob Agents. 2003;21:96-101. doi: 110.1016/s0924-8579(1002)00364-00363.

14. Basheti IA, Nassar R, Barakat M, et al. Pharmacists' readiness to deal with the coronavirus pandemic: Assessing awareness and perception of roles. Res Social Adm Pharm. 2020;23:30418-30416.

15. Sokolow LZ, Patel A, Koonin LM, Graitcer SB. Scripted Surge Pharmacy Pandemic Exercise: Testing Vaccine Administration and Antiviral Dispensing. Health Secur. 2018;16:262-273. doi: 210.1089/hs.2018.0031.

16. Rosenfeld LA, Etkind P, Grasso A, Adams AJ, Rothholz MC. Extending the reach: local health department collaboration with community pharmacies in Palm Beach County, Florida for H1N1 influenza pandemic response. J Public Health Manag Pract. 2011;17:439-448. doi: 410.1097/PHH.1090b1013e31821138ae.

17. SteelFisher GK, Benson JM, Caporello H, et al. Pharmacist Views on Alternative Methods for Antiviral Distribution and Dispensing During an Influenza Pandemic.Health Secur. 2018;16:108-118. doi: 110.1089/hs.2017.0068. Epub 2018 Mar 1023. 
18. Nazar Z, Nazar H. Exploring the experiences and preparedness of humanitarian pharmacists in responding to an emergency-response situation. Res Social Adm Pharm. 2020;16:90-95. doi: 10.1016/j.sapharm.2019.1003.1146. Epub 2019 Mar 1031.

19. Song Z, Hu Y, Zheng S, Yang L, Zhao R. Hospital pharmacists' pharmaceutical care for hospitalized patients with COVID-19: Recommendations and guidance from clinical experience. Res Social Adm Pharm. 2020;3:30314-30314.

20. Khan Z, Muhammad K, Ahmed A, Rahman H. Coronavirus outbreaks: prevention and management recommendations. Drugs \& Therapy Perspectives. 2020:1-3.

21. Gross AE, MacDougall C. Roles of the clinical pharmacist during the COVID-19 pandemic. Journal of the American colledge of clinical pharmacy 2020:1-3.

22. Miller S, Patel N, Vadala T, Abrons J, Cerulli J. Defining the pharmacist role in the pandemic outbreak of novel H1N1 influenza. J Am Pharm Assoc (2003). 2012;52:763-767. doi: 710.1331/JAPhA.2012.11003.

23. McCourt E. Improving pharmacist involvement in pandemic influenza planning and response in Australia. Found at https://ahha.asn.au/system/files/docs/publications/summary_deeble_institute_issues_brief_no._25.pdf. Accessed 4, 2020. Australian Healthcare and Hospital Association.2018.

24. Zheng SQ, Yang L, Zhou PX, Li HB, Liu F, Zhao RS. Recommendations and guidance for providing pharmaceutical care services during COVID-19 pandemic: A China perspective. Res Social Adm Pharm. 2020;26:30284-30289.

25. WHO Coronavirus Disease (COVID-19) Dashboard. Accessed on May-2020. Found at https://covid19.who.int/.

26. Hernandez-Garcia I, Gimenez-Julvez T. Assessment of Health Information About COVID-19 Prevention on the Internet: Infodemiological Study. JMIR Public Health Surveill. 2020;6:e18717. doi: $18710.12196 / 18717$.

27. Karasneh R, Al-Azzam S, Muflih S, Soudah O, Hawamdeh S, Khader Y. Media's effect on shaping knowledge, awareness risk perceptions and communication practices of pandemic COVID-19 among pharmacists. Res Social Adm Pharm. :.10.1016/j.sapharm.2020.1004.1027.

28. Day M. Covid-19: ibuprofen should not be used for managing symptoms, say doctors and scientists. 2020;368:m1086.

29. FDA cautions against use of hydroxychloroquine or chloroquine for COVID-19 outside of the hospital setting or a clinical trial due to risk of heart rhythm problems. Cited on 42020/ Available from: https://www.fda.gov/drugs/drug-safety-and-availability/fda-cautions-against-usehydroxychloroquine-or-chloroquine-covid-19-outside-hospital-setting-or.

30. Sanders JM, Monogue ML, Jodlowski TZ, Cutrell JB. Pharmacologic Treatments for Coronavirus Disease 2019 (COVID-19): A Review. Jama. 2020;13.

31. Le Bourgeois M, Ferroni A, Leruez-Ville M, et al. Nonsteroidal Anti-Inflammatory Drug without Antibiotics for Acute Viral Infection Increases the Empyema Risk in Children: A Matched Case-Control Study. J Pediatr.2016;175:47-53.e3.:10.1016/j.jpeds.2016.1005.1025. Epub 2016 Jun 1020.

32. Capuano A, Scavone C, Racagni G, Scaglione F. NSAIDs in patients with viral infections, including Covid-19: victims or perpetrators? Pharmacol Res.2020;29:104849.

33. Alobaedi $\mathrm{OH}$, Talib WH, Basheti IA. Antitumor effect of thymoquinone combined with resveratrol on mice transplanted with breast cancer. Asian Pac J Trop Med.2017;10:400-408. doi: 410.1016/j.apjtm.2017.1003.1026. Epub 2017 Apr 1017. 
34. Lima CKT, Carvalho PMM, Lima I, et al. The emotional impact of Coronavirus 2019-nCoV (new Coronavirus disease). Psychiatry Res.2020;287:112915.:10.1016/j.psychres.2020.112915.

35. Walton M, Murray E, Christian MD. Mental health care for medical staff and affiliated healthcare workers during the COVID-19 pandemic. Eur Heart J Acute Cardiovasc Care. 2020;28:2048872620922795.

36. FIP. Coronavirus SARS-CoV-2 / COVID-19 pandemic: Information and Guidelines for Pharmacists and the Pharmacy Workforce Vol 2020. The Netherlands: International Pharmaceutical Federation 2020.

37. Nazer LH, Tuffaha H. Health Care and Pharmacy Practice in Jordan. Can J Hosp Pharm.2017;70:150155. doi: 110.4212/cjhp.v4270i4212.1649. Epub 2017 Apr 4228.

38. Tyrer S, Heyman B. Sampling in epidemiological research: issues, hazards and pitfalls.BJPsych Bull. 2016;40:57-60. doi: 10.1192/pb.bp.1114.050203.

39. Nelson LM, Simard JF, Oluyomi A, et al. US Public Concerns About the COVID-19 Pandemic From Results of a Survey Given via Social Media. JAMA Intern Med.2020;7.

40. Bates I, Bader LR, Galbraith K. A global survey on trends in advanced practice and specialisation in the pharmacy workforce. Int J Pharm Pract.2020;28:173-181. doi: 110.1111/ijpp.12611.

Figure legends

Figure 1. Study process showing the five sections of the online survey used in this study.

Figure 2. Study participants $(n=2589)$ awareness about epidemics/pandemics and updates about coronavirus treatment based on region.

Figure 3. Sources of information about coronavirus treatment among the study participants $(\mathrm{n}=2589)$.

Figure 4. Study participants' perceptions about the current role of the faculties of pharmacies/educational institutes, pharmacists' associations and societies, and pharmacists' role to deal with epidemics/pandemics and the coronavirus pandemic specifically $(\mathrm{n}=2589)$.

\section{Hosted file}

Tables.docx available at https://authorea.com/users/310289/articles/458795-pharmacistsawareness-of-covid-19-and-perceptions-of-their-roles-barriers-and-roles-of-policymakersexploring-the-middle-east-and-north-africa-mena

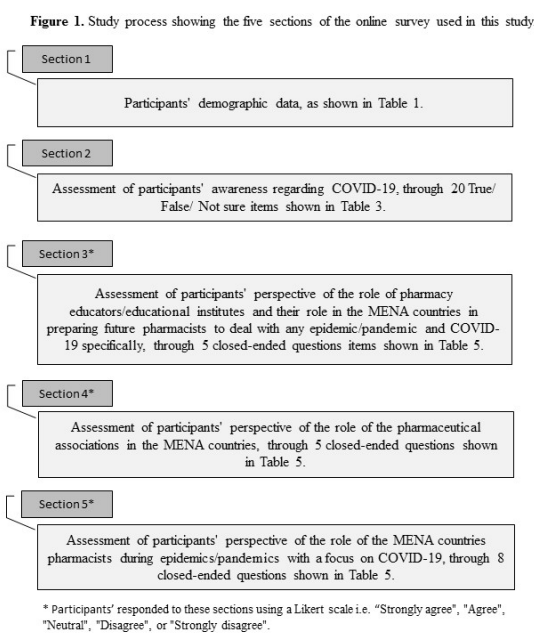


Figure 2. Study patticipants (n-2589) awareness about epidemics/pandemics and updates about coronavirus treatment based on region.

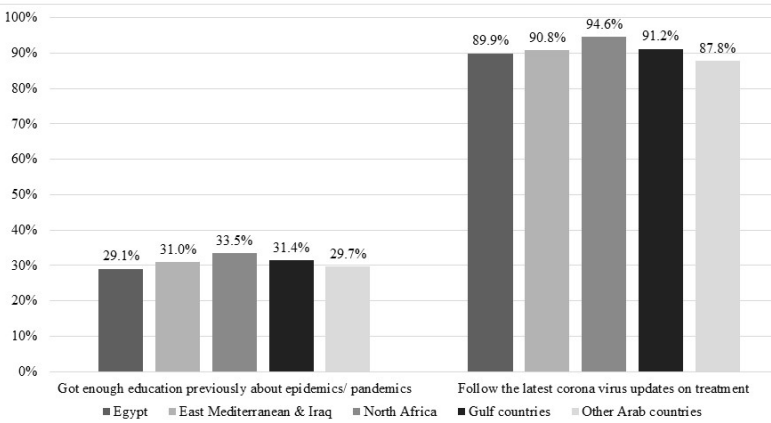

Figure 3. Sources of information about coronavinus treatment among the study participants (n=2589)

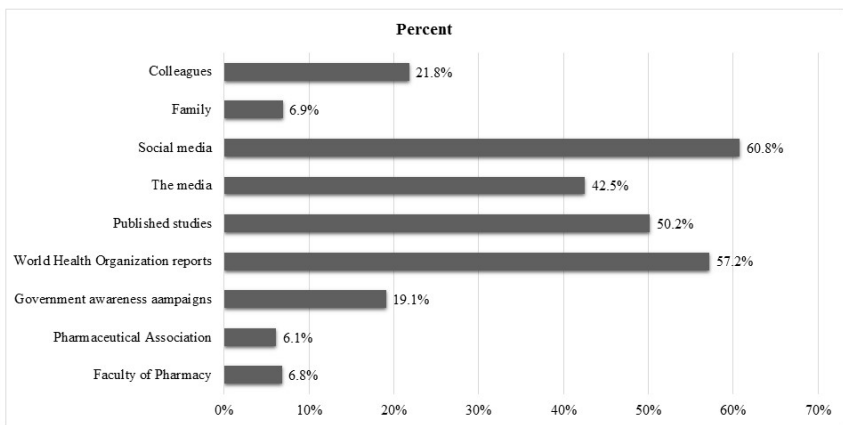

Figure 4. Study patticipants' perceptions about the current role of the facultics of pharmacies/cducational institutcs, phamacists' associations and socictics,

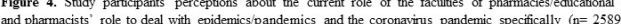

Stronly agree/agree

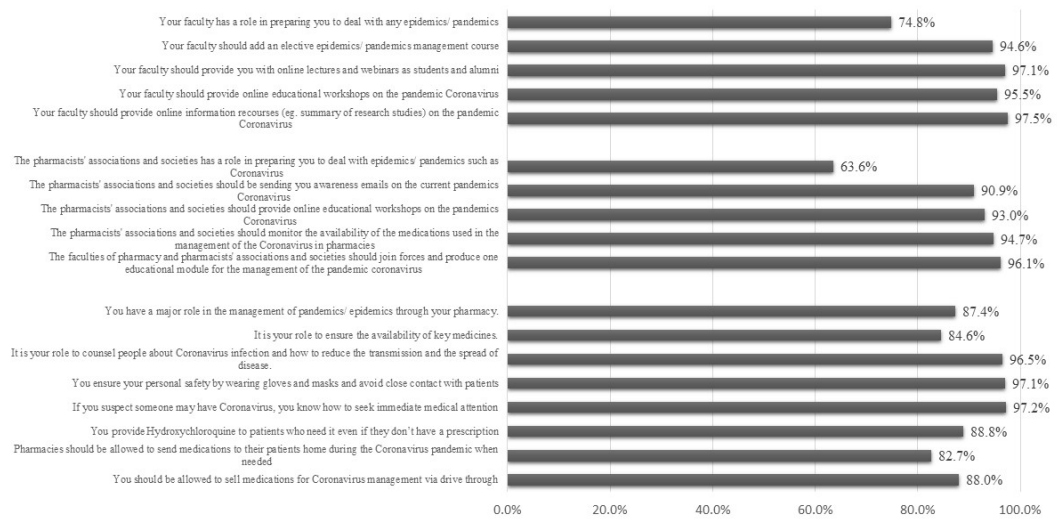

